\title{
Towards Fog-Free In-Vehicle Vision Systems through Contrast Restoration
}

\author{
Nicolas Hautière, Jean-Philippe Tarel \\ LCPC \\ 58 boulevard Lefebvre \\ 75732 Paris Cedex 15, France \\ hautieredlcpc.fr, tarelelcpc.fr
}

\author{
Didier Aubert \\ LIVIC - LCPC / INRETS \\ 14 route de la Minière \\ 78000 Versailles, France \\ auberteinrets.fr
}

\begin{abstract}
In foggy weather, the contrast of images grabbed by invehicle cameras in the visible light range is drastically degraded, which makes the current applications very sensitive to weather conditions. An onboard vision system should take fog effects into account. The effects of fog varies across the scene and are exponential with respect to the depth of scene points. Because it is not possible in this context to compute the road scene structure beforehand contrary to fixed camera surveillance, a new scheme is proposed. Weather conditions are first estimated and then used to restore the contrast according to a scene structure which is inferred a priori and refined during the restoration process. Based on the aimed application, different algorithms with increasing complexities are proposed. Results are presented using sample road scenes under foggy weather and assessed by computing the contrast before and after restoration.
\end{abstract}

\section{Weather and Onboard Vision}

Under degraded weather conditions, the contrast of images which are grabbed by a classical in-vehicle camera in the visible light range is drastically degraded, which makes current in-vehicle applications relying on such sensors very sensitive to weather conditions. An in-vehicle vision system should take fog effects into account to be more reliable. A first solution is to adapt the operating thresholds of the system or to deactivate it momentarily if these thresholds have been surpassed. A second solution is to remove fog effects from the image beforehand. Unfortunately, haze effects vary across the scene. They are exponential with respect to the depth of scene points. Consequently, space invariant filtering techniques cannot be used directly to adequately remove weather effects from images. A judicious approach is to detect the weather conditions so as to estimate the decay in the image and then to remove it.
The majority of sensors dedicated to measuring visibility distances (scatterometer, transmissometer) are expensive to operate and quite often complicated to install and calibrate correctly. Moreover, this type of equipment cannot easily be placed onboard a vehicle. Using a camera does not entail such obstacles. Bush [5] and Kwon [10] relied upon a fixed camera placed above the roadway for the purpose of measuring visibility distances. However, systems that meet this purpose with an onboard camera are encountered less frequently. Pomerleau [22] estimates visibility by means of measuring the contrast attenuation of road markings at various distances in front of a moving vehicle. Hautière et al. [9] detect the presence of daytime fog and estimate the meteorological visibility distance. An extension of their method using stereovision is presented in [8].

Methods which restore image contrast under bad weather conditions are encountered more often in the literature. Unfortunately, they all have rather constraints too strong to be used onboard a moving vehicle. Some techniques require prior information about the scene [21]. Others require dedicated hardware in order to estimate the weather conditions [25]. Some techniques rely only on the acquired images and exploit the atmospheric scattering to obtain the range map of the scene $[19,6]$. This range map is then used to adequately restore the contrast. However, these methods require fog conditions to change between image acquisitions. Techniques based on polarization can also be used to reduce haziness in the image [23]. Unfortunately, they require two differently filtered images of the same scene. Some techniques assume a flat world scene like [20]. The authors compute the extinction coefficient of fog and assume a flat world seen from a forward-looking airborne camera. However, they approximate the distribution of radiances in the image with a simple Gaussian with known variance. Finally, it is proposed in [17] to restore the contrast of more complex scenes. However, the user must manually specify a location for sky region, vanishing point and an approxi- 
mation of distance distribution in the image.

In this paper, we propose to restore automatically the contrast of images grabbed by an in-vehicle camera. Because it is not possible in this context to compute the road scene structure beforehand, we propose a scheme quite opposite to the one proposed in [19]. Thus, weather conditions are first estimated and used to restore the contrast according to a road scene structure, which is inferred a priori and then refined during the contrast restoration process. According to the aimed in-vehicle application, different algorithms with increasing complexities are proposed. Results are presented using sample frames from three different video clips of road scenes under foggy weather.

The paper is organized as follows. We first present a model of fog visual effects. Then, a technique estimating the extinction coefficient of the atmosphere in the current image from a single camera is presented. Once the weather condition is known, a contrast restoration principle is proposed with its associated tools. Finally, we present three different applications implementing the principle presented in the previous section. The assessment of the principle is done by first computing the pixels which have been distorted during the contrast restoration process and second computing the contrast of the images before and after scene restoration.

\section{Fog Effects on Vision}

The literature on the interaction of light with the atmosphere has been written over more than two centuries [4, 2]. Different reviews on the subject are for a long time available in the literature $[15,16,14]$ and still serve as reference for more recent works in computer vision $[6,18,9]$. In this section, selected results dealing with fog effects on vision are presented.

\subsection{Optical Properties of Fog}

In fog, a proportion of the light is scattered by water droplets and thus deviates from its path. Because the absorption of visible light by water droplets proves to be negligible [3], the scattering and extinction coefficients tends to be interchangeable. The relation tying the incident luminous flux $\Phi_{0}$ to the transmitted flux $\Phi_{T}$ is known as BeerLambert's law and is expressed as follows:

$$
\Phi_{T}=\Phi_{0} e^{-\beta d}
$$

where $d$ is the distance and $\beta$ is the atmospheric extinction coefficient of the medium and is related to droplet size distribution of fog.

\subsection{Visual Properties of Fog}

The attenuation of luminance through the atmosphere was studied by Koschmieder [15], who derived an equation

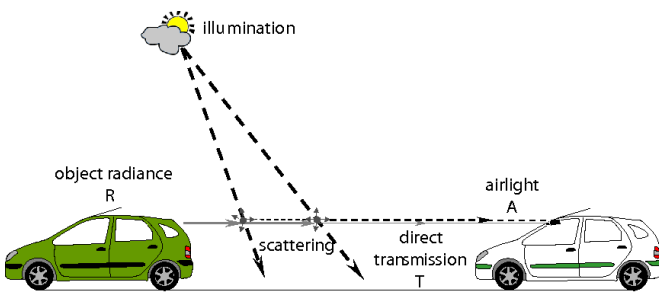

Figure 1. Fog or haze luminance are due to the scattering of daylight. Light coming from the sun and scattered by atmospheric particles towards the camera is the airlight $A$. It increases with the distance. The light emanating from the object $R$ is attenuated by scattering along the line of sight. Direct transmission $T$ of $R$ decreases with distance.

relating the apparent luminance or radiance $L$ of an object located at distance $d$ to the luminance $L_{0}$ measured close to this object:

$$
L=L_{0} e^{-\beta d}+L_{\infty}\left(1-e^{-\beta d}\right)
$$

This expression indicates that the luminance of the object seen through fog is attenuated in $e^{-k d}$ (Beer-Lambert law); it also reveals a luminance reinforcement of the form $L_{\infty}\left(1-e^{-\beta d}\right)$ resulting from daylight scattered by the slab of fog between the object and the observer, also named airlight. $L_{\infty}$ is the atmospheric luminance. In the presence of fog, it is also the background luminance on which the target can be detected. The previous equation may then be written as follows:

$$
L-L_{\infty}=\left(L_{0}-L_{\infty}\right) e^{-\beta d}
$$

On the basis of this equation, Duntley developed a contrast attenuation law [15], stating that a nearby object exhibiting contrast $C_{0}$ with the background will be perceived at distance $d$ with the following contrast:

$$
C=\left(\left(L-L_{\infty}\right) / L_{\infty}\right) e^{-\beta d}=C_{0} e^{-\beta d}
$$

This expression serves to base the definition of a standard dimension called "meteorological visibility distance" $V_{m e t}$, i.e. the greatest distance at which a black object $\left(C_{0}=\right.$ -1 ) of a suitable dimension can be seen in the sky on the horizon, with the threshold contrast set at 5\% [1]. It is thus a standard dimension that characterizes the opacity of a fog layer. This definition yields the following expression:

$$
V_{\text {met }}=-\frac{1}{\beta} \log (0.05) \simeq \frac{3}{\beta}
$$

\subsection{Camera Response}

Let us denote $f$ the camera response function, which models the mapping from scene luminance to image intensity by the imaging system, including optic as well as electronic parts [7]. With the notations of Fig. 1, the intensity $I$ 


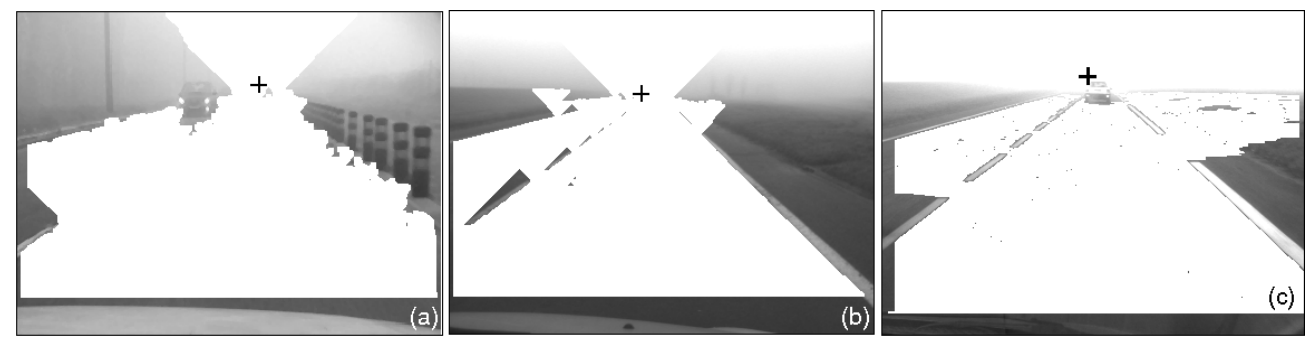

Figure 2. Results of the region growing on the test images: the road and the sky are partially segmented and are painted white. The black cross indicates the position of the vanishing point: its horizontal location $u_{h}$ is approximated by the middle of the area obtained by the region growing on the horizon line $v_{h}$. This latter value is obtained by a priori sensor calibration.

of a pixel is the result of $f$ applied to the sum of the airlight $A$ and the direct transmission $T$, i.e:

$$
I=f(L)=f(T+A)
$$

In this work, we assume that the conversion process between incident energy on the CCD sensor and the intensity in the image is linear. This is generally the case for short exposure times, because it prevents CCD array to be saturated. Furthermore, short exposure times (1 to $4 \mathrm{~ms}$ ) are used on in-vehicle cameras to reduce the motion blur. This assumption can thus be considered as valid and (6) becomes:

$$
\begin{aligned}
I & =f(T)+f(A)=f\left(L_{0} e^{-\beta d}\right)+f\left(L_{\infty}\left(1-e^{-\beta d}\right)\right) \\
& =f\left(L_{0}\right) e^{-\beta d}+f\left(L_{\infty}\right)\left(1-e^{-\beta d}\right) \\
& =R e^{-\beta d}+A_{\infty}\left(1-e^{-\beta d}\right)
\end{aligned}
$$

where $R$ is the intrinsic intensity of the pixel, i.e. the intensity corresponding to the intrinsic luminance value of the corresponding scene point and $A_{\infty}$ is the background sky intensity.

\section{Estimation of the Extinction Coefficient of Fog}

In this section, a method to compute the extinction coefficient $\beta$ using a single camera behind the vehicle windshield is recalled from $[9,12]$.

\subsection{Flat World Hypothesis}

In the image plane, the position of a pixel is given by its $(u, v)$ coordinates. The coordinates of the optical center projection in the image are designated by $\left(u_{0}, v_{0}\right) . H$ denotes the height of the camera, $\theta$ the angle between the optical axis of the camera and the horizontal, and $v_{h}$ the horizon line. The intrinsic parameters of the camera are its focal length $f_{l}$, and the horizontal size $t_{p u}$ and vertical size $t_{p v}$ of a pixel. We have also made use herein of $\alpha_{u}=\frac{f_{l}}{t_{p u}}$ and $\alpha_{v}=\frac{f_{l}}{t_{p v}}$, and have typically considered: $\alpha_{u} \approx \alpha_{v}=\alpha$. The hypothesis of a flat road is adopted, which makes it possible to associate a distance $d$ with each line $v$ of the image:

$$
d=\frac{\lambda}{v-v_{h}} \text { if } v>v_{h}, \text { where } \lambda=\frac{H \alpha}{\cos ^{2} \theta}
$$

\subsection{Recovery of Koschmieder's Law Parameters}

Following a variable change from $d$ to $v$ based on (8), (7) then becomes:

$$
I=R-\left(R-A_{\infty}\right)\left(1-e^{-\beta \frac{\lambda}{v-v_{h}}}\right)
$$

By twice taking the derivative of $I$ with respect to $v$, one obtains the following:

$$
\frac{d^{2} I}{d v^{2}}=\beta \varphi(v) e^{-\beta \frac{\lambda}{v-v_{h}}}\left(\frac{\beta \lambda}{v-v_{h}}-2\right)
$$

where $\varphi(v)=\frac{\lambda\left(R-A_{\infty}\right)}{\left(v-v_{h}\right)^{3}}$. The equation $\frac{d^{2} I}{d v^{2}}=0$ has two solutions. The solution $\beta=0$ is of no interest. The only useful solution is given in (11):

$$
\beta=\frac{2\left(v_{i}-v_{h}\right)}{\lambda}
$$

where $v_{i}$ denotes the position of the inflection point of $I(v)$. In this manner, the parameter $\beta$ of Koschmieder's law is obtained once $v_{i}$ is known. Finally, thanks to $v_{i}, v_{h}$ and $\beta$ values, the values of the other parameters of (7) are deduced through use of $I_{i}$ and $\frac{d I}{d v}{ }_{v=v_{i}}$, which are respectively the values of the function $I$ and its derivative in $v=v_{i}$ :

$$
\begin{aligned}
R & =I_{i}-\left(1-e^{-\beta d_{i}}\right) \frac{\left(v_{i}-v_{h}\right)}{2 e^{-\beta d_{i}}} \frac{d I}{d v}{ }_{\mid v=v_{i}} \\
A_{\infty} & =I_{i}+{\frac{\left(v_{i}-v_{h}\right)}{2}}_{\frac{d I}{d v}}{ }_{\mid v=v_{i}}
\end{aligned}
$$

where $R$ is the mean intrinsic intensity of the road surface.

\subsection{Implementation}

\subsubsection{Estimation of the inflection point}

To estimate the parameters of (7), the median intensity on each line of a vertical band is estimated and an inflection point is detected. So as to be in accordance with 

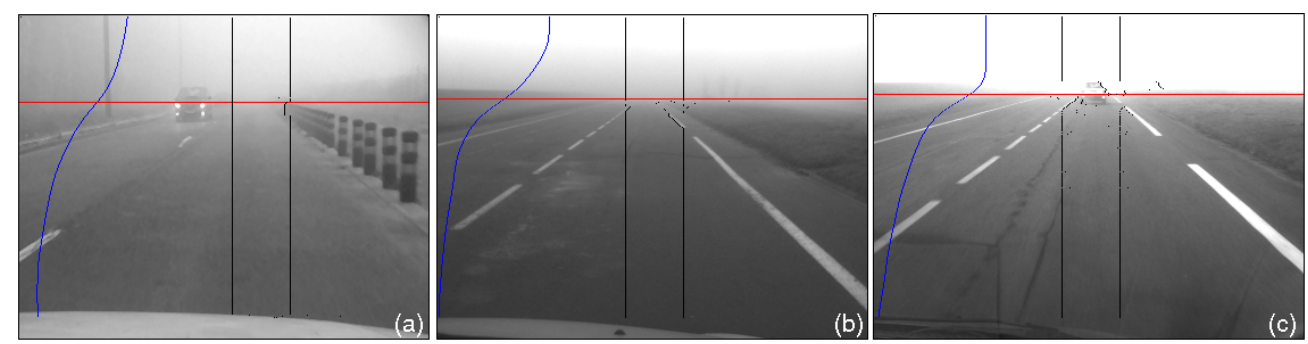

Figure 3. Black segments: measurement bandwidth. Curve on the left: vertical variation of intensity within the bandwidth. Horizontal line: image line representative of the meteorological visibility distance.

Koschmieder's law assumptions, this band should only take into account a homogeneous area and the sky. Thus, a region within the image that displays minimal line-to-line gradient variation when browsed from bottom to top is identified thanks to a region growing process, illustrated in Fig. 2. A vertical band is then selected in the detected area. Finally, taking the median intensity of each segment, yields the vertical variation of the intensity of the image and the position of the inflection point.

\subsubsection{Estimation of the horizon line position}

To obtain the values of the parameters of (7), the position of the horizon line must be estimated. It can be estimated by means of the pitching of the vehicle when an inertial sensor is available, but is generally estimated by an additional image processing. Generally, this type of processing seeks to intersect the vanishing lines in the image. However, under foggy weather, the vanishing lines are only visible close to the vehicle. It is thus necessary to extrapolate the position of the horizon line through the fog. Consequently, this kind of process is prone to a significant standard deviation and, for the moment, we use the a priori sensor calibration.

Having now the vertical positions of both the inflection point and the horizon line, the parameters of (7) can be recovered and the position of the image line representative of the meteorological visibility distance is deduced. Fig. 3 illustrates the process.

\section{Tools for Contrast Restoration}

In this section, we present three tools for contrast restoration: a restoration method based on Koschmieder's law, a scene depth modeling and an image quality attribute used to evaluate the quality of the restoration.

\subsection{Restoration Method}

In this section, we describe a simple method to restore scene contrast from an image of a foggy scene. Let us consider a pixel with known depth $d$. Its intensity $I$ is given by (7). $\left(A_{\infty}, \beta\right)$ characterizes the weather condition and is estimated thanks to section 3. Consequently, contrary to (12),
$R$ can be estimated directly for all scene points from:

$$
R=I e^{\beta d}+A_{\infty}\left(1-e^{\beta d}\right)
$$

To correctly restore the scene contrast, the remaining problem is the estimation of the depth $d$ of each pixel.

\subsection{Scene Depth Modeling}

If we assume that the whole scene is flat, (8) is enough to estimate the depth of scene points and consequently to restore the contrast of the image. Unfortunately, in this way, all the contrast of all vertical objects of the scene (other vehicles, trees...) will be falsely restored. In order to estimate the depth of scene points, [17] proposes some depth heuristics to interactively restore contrast. In this aim, the user selects a region of the sky to obtain the sky intensity $A_{\infty}$ and inputs the approximate location of the vanishing point of the image along the direction of increasing distances in the image. Here, the sky region and the vanishing point location are automatically extracted, thanks to the region growing process described in paragraph 3.3.1. Thus, the sky region is assumed to be the region of the image above the horizon line where intensity is higher than the intensity taken at the horizon line. The coordinate system is given in Fig. 4(a). The horizontal location of the vanishing point $u_{h}$ is approximated by the center of the area obtained by the region growing on the horizon line. For our test images, the location of the vanishing point is marked with a black cross in Fig. 2. Then, we propose to use a depth heuristic in conjunction with the flat world assumption. Following (8), we use the flat world assumption for points belonging to the road surface, except for big distances which are clipped using parameter $c$ to reduce the modeling errors (in particular, flat world and non flat world are mixed near the horizon line):

$$
d_{1}= \begin{cases}\frac{\lambda}{v-v_{h}} & \text { if } v-v_{h}>c \\ \frac{\lambda}{c-v_{h}} & \text { if } 0<v-v_{h} \leq c\end{cases}
$$

Then, we use a depth heuristic issued from [17] to model the depth of points not belonging to the road surface:

$$
d_{2}=\frac{\kappa}{u-u_{h}} \text { or } \frac{\kappa}{\sqrt{\left(u-u_{h}\right)^{2}+\left(v-v_{h}\right)^{2}}}
$$



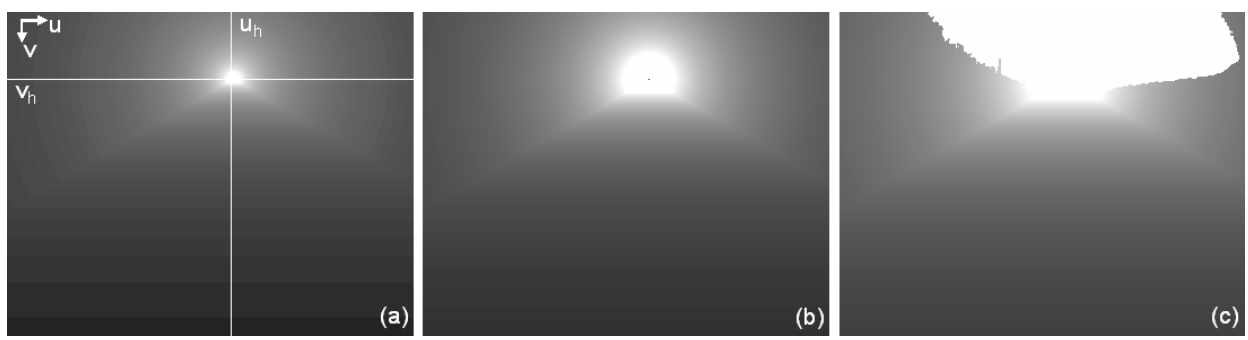

Figure 4. Construction of the depth modeling for the test image of Fig. 3a. (a) a cylindrical scene where a small value of $\kappa$ is assumed. (b) a cylindrical scene where a bigger value of $\kappa$ is assumed, resulting in a larger road surface than in Fig. 4a. (c) The sky region has been added.

where $\kappa \geq \lambda$. The first heuristic can be used to model vertical objects like building in urban streets. The second heuristic is better suited to model cylindrical scenes like rural roads. The final depth $d$ of a pixel $(u, v)$ which does not belong to the sky region is finally:

$$
d=\min \left(d_{1}, d_{2}\right)
$$

In these depth heuristics, the value of $\kappa$ controls the relative importance of the flat world against the vertical surroundings. Fig. 4 illustrates the depth modeling for the test image of Fig. 3(a). In order to restore correctly the contrast, the remaining problem is to determine the optimal values of $\kappa$ and $c$.

\subsection{Image Quality Attribute}

According to (14), if the depth of a point is overestimated, its restored intensity becomes null or negative, which creates some artefacts in the restored image and alters its visual quality. During the restoration process, the challenging problem is thus to detect the degradations. We have to cope with a blind image quality assessment problem. Such a problem is generally encountered in the image compression area, where quality attributes are generally based on blur, noise and compression artefacts [24]. These quality attributes do not match with our problem. We propose another quality attribute better suited for our problem: $Q$ is the norm of the local normalized correlation between the original image $I$ and the restored image $R$ :

$$
Q=\|h(I, R)\|
$$

where $h$ denotes a normalized correlation formula. Currently, we use the $Z N C C$ criteria, that is to say:

$$
h(I, R)=H\left[\frac{\sum_{i}(I(x+i)-\bar{I})(R(x+i)-\bar{R})}{\sqrt{\sum_{i}(I(x+i)-\bar{I})^{2} \sum_{i}(R(x+i)-\bar{R})^{2}}}\right]
$$

where $H$ denotes the Heaviside function, $\bar{I}$ and $\bar{R}$ the means of pixel intensities for the neighborhoods of $I$ and $R$ centered at $x$. Indeed, we can assume that within small neighborhoods containing visual information, the depth of the points are not very different. Consequently, the normalized correlation score between the original and the restored versions of a neighborhood should remain high. A decreasing normalized correlation means that the content of the original and restored neighborhoods differ. Criteria (18) can be used to automatically estimate $\kappa$ and $c$, as explained in the following.

\section{In-Vehicle Applications}

Depending on the developed in-vehicle application, the principles that have been presented in the previous section can be used and combined differently to restore the contrast of images. In this section, three applications with an increasing complexity are detailed.

\subsection{Contrast Restoration of the Road Surface}

The first application consists in restoring the road surface only. Vertical objects are ignored and the values of $\kappa$ and $c$ are chosen a priori. In this way, vertical objects may be distorted. This is adequate to enhance the detection of road markings under foggy weather. Used in conjunction with a driving assistance relying on lane markings extraction, it can lead to a better prevention of road departures [13]. An example of such a contrast restoration is given in Fig. 5. The distorted pixels are shown in Fig. 8(a). As expected, the contrast of the road surface is restored. It is interesting to notice that the contrast of the trees in the background of the image is enhanced. One may distinguish a car at the bottom of those trees. These facts are confirmed by the computation of the local contrasts above 5\% (see section 6.1).

\subsection{Contrast Enhancement of the Road Scene}

A second application consists in taking into account the vertical objects that are present in the road scene including the borders and the objects on the road itself. The method aims at restoring the contrast of the road surface, while enhancing contrast on vertical objects without distorting them too much. Thus, we seek the best scene depth model which maximizes the contrast and minimizes the number of dis- 


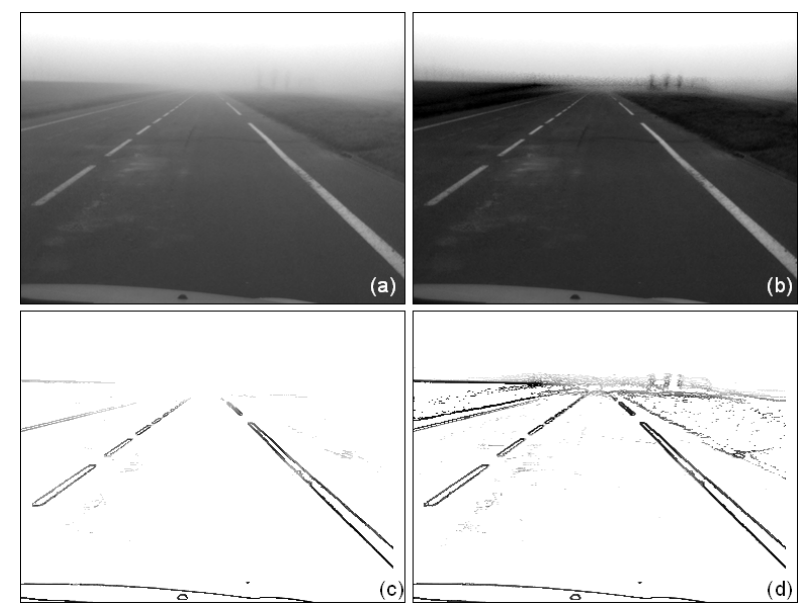

Figure 5. Example of contrast restoration of a road surface. (a) Original image. (b) Image with restored contrast. (c) Contrasts greater than $5 \%$ on the original image and (d) on the restored image. Used parameters: $\lambda=700, \kappa=6 \lambda, c=12, A_{\infty}=220$. Estimated parameters: $v_{h}=69, \beta=0.026, V_{\text {met }} \approx 116 \mathrm{~m}$.

torted pixels, i.e. the optimal values of $\kappa$ and $c$. The problem can be formulated as a minimization process:

$$
\left(\kappa^{*}, c^{*}\right)=\underset{\substack{\kappa>1 \\ c>0}}{\operatorname{argmax}}(Q(\kappa, c)+\kappa-c+)
$$

In this application, the $\ell_{1}$ norm is used in (18) for evaluating the quality $Q$. Currently, (20) is solved using Powell's method. An example is given in Fig. 6. The distorted pixels are shown in Fig. 8(b). When the first car and the road surroundings are partially distorted, the contrast is well restored on the second vehicle, which is now visible within the white circle. This is confirmed by Fig. 6(d), which shows that the second car now has a contrast greater than $5 \%$. Furthermore, the method copes with curved road by decoupling the slope estimation of $\kappa$ of the left and right border planes.

\subsection{Iterative Contrast Restoration}

The previous method enhances but sometimes distorts the contrast of vertical objects on the road. By merging an object (sign, vehicle, etc.) detection algorithm within the restoration process, contrast can be restored on both the detected objects and the road surface, and enhanced on the borders. The method becomes iterative and alternates restoration and detection processes. Its principle is first to restore the contrast using a small value of $\kappa$ (typically $\kappa=1$ ) and then to progressively increase its value until the restored image gets degraded. In this way, the flat world is extended as much as possible according to the vertical objects which are present in the scene. Then, to temporarily stop the restoration, we look at the strong variations of the quality attribute $Q$ with respect to the value of $\kappa$. Thus, we

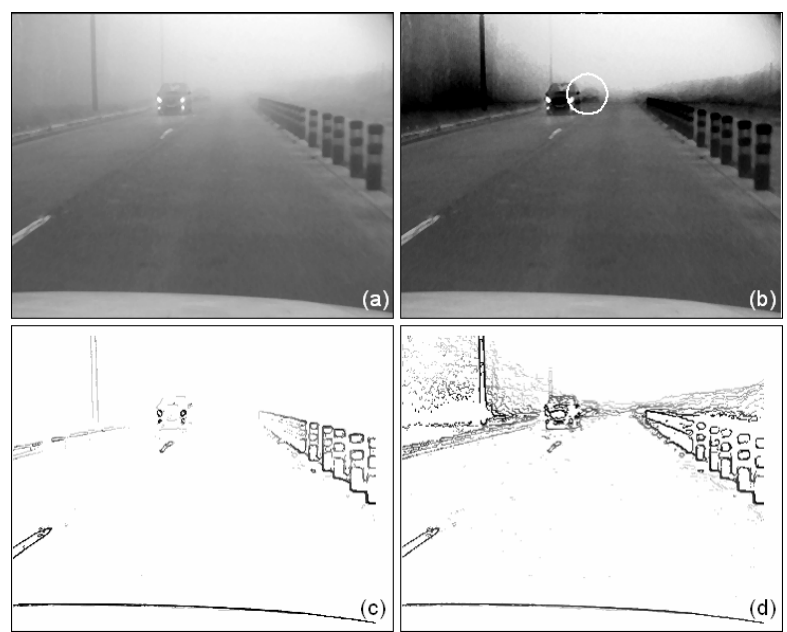

Figure 6. Example of contrast enhancement of a road scene. (a) Original image. (b) Image with restored contrast. (c) Contrasts greater than $5 \%$ on the original image and (d) on the restored image. Used parameters: $\lambda=700$. Estimated parameters: $v_{h}=70$, $\beta=0.034, V_{\text {met }} \approx 87 m, c^{*}=14, \kappa^{*}=8.6 \lambda A_{\infty}=208$.

search $\kappa^{*}$, such as:

$$
\frac{\partial Q}{\partial \kappa}\left(\kappa^{*}\right)>t
$$

In this application, the $\ell_{\infty}$ norma is used for estimating $Q$. If a strong variation is detected, objects belonging to the desired class are searched in the image. If objects are found, their positions are estimated and their contrast correctly restored thanks to a bounding box fitted around them. Then, the restoration restarts and avoids the different bounding boxes. Like for the previous application, the estimation of the left and right border planes is decoupled. An example is given in Fig. 7 within a vehicle detection process using [11] which allows to cope with non flat road geometry. On this scene, a bad contrasted vehicle is in front of the equipped vehicle. The result of the initial contrast restoration process is given in Fig. 7(b). It is stopped at $\kappa=1.25 \lambda$ (cf. left peak of Fig. 9). Because contrast has been enhanced on the vehicle, it can be detected more easily by a dedicated method and a bounding box can be fitted around it, as illustrated in Fig. 7(c). Finally, contrast restoration restarts and stops at $\kappa=4.25 \lambda$ (cf. right peak of Fig. 9). The final image with the contrast of the vehicle properly restored and not saturated (contrary to Fig. 7(b)) is given in Fig. 7(d). The improvement of contrast is shown in Figs. 7(e)(f).

\section{Checking Contrast Improvement}

To check contrast improvement, we first detect the local distorted pixels using $Q$ values (cf. Fig. 8). Then, in the following, we compute the local contrasts $C(s)$ before and after the contrast restoration. Following CIE recommenda- 


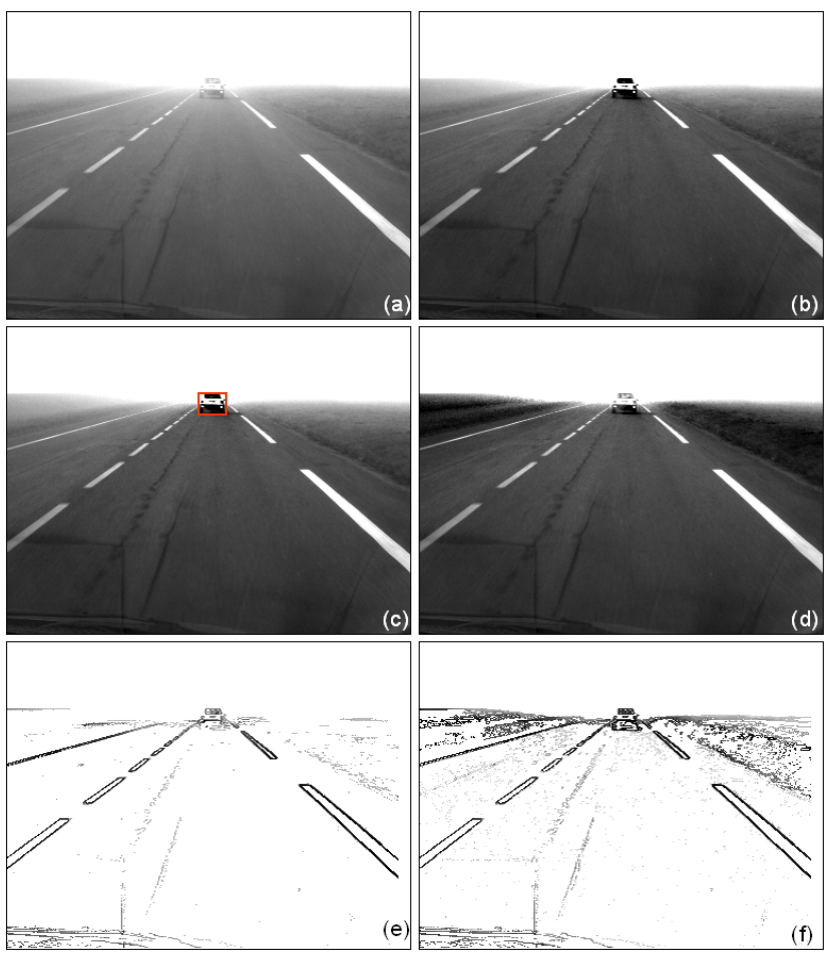

Figure 7. Example of iterative contrast restoration of a road scene. (a) Original image. (b) Restored image after initial contrast restoration $\kappa_{1}=1.25 \lambda$. (c) Result of vehicle detection using the first restored image. (d) Final restored image with the vehicle properly restored $\kappa_{2}=4.25 \lambda$. (e) Contrasts greater than $5 \%$ on the original image and (f) on the restored image. Used parameters: $\lambda=700, c=1, t=0.01$. Estimated parameters: $v_{h}=60$, $\beta=0.05, V_{\text {met }} \approx 58 \mathrm{~m}, A_{\infty}=250$.

tions [1], a visibility threshold of $5 \%$ is used. $C(s)$ measures linear contrast enhancement, whereas $Q$ is invariant to linear contrast variations but not to contrast saturations.

\subsection{Computation of local contrasts above $5 \%$}

We adapted Köhler's binarization technique [?] in order to measure the local contrasts of images. A pair of pixels $\left(x, x_{1}\right)$ is said to be separated by $s$ if two conditions are met. First, $x_{1} \in N_{4}(x)$. Second, the condition $\min \left(I(x), I\left(x_{1}\right)\right) \leq s<\max \left(I(x), I\left(x_{1}\right)\right)$ is respected.
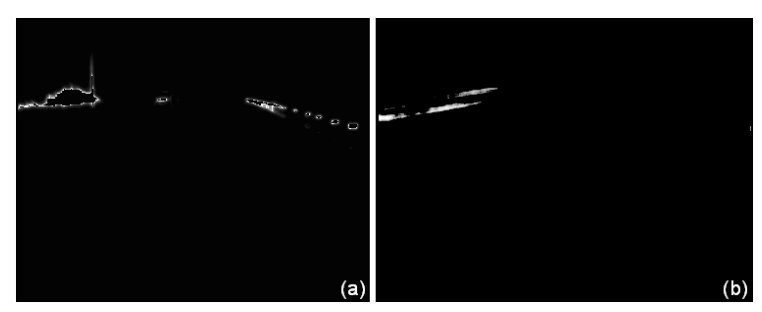

Figure 8. Distorted pixels on the restored images (a) distortion computed on Fig.5(d) and (b) on 6(d). As expected, only vertical objects are distorted.

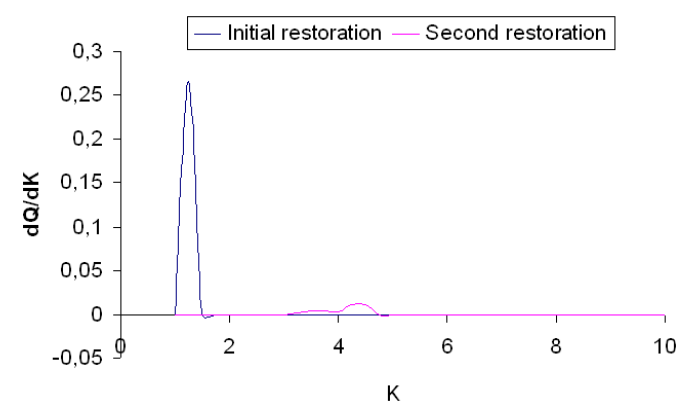

Figure 9 . Variation of the image quality attribute $\left(\ell_{\infty}\right.$ norm) with respect to the value of $\kappa$. Blue curve: initial restoration stopped at $\kappa_{1}=1.25 \lambda$. Pink curve: final restoration stopped at $\kappa=4.25 \lambda$.

Let $F(s)$ be the set of all couples $\left(x, x_{1}\right)$ separated by $s$. With these definitions, for every value of $s$ belonging to [0,255], $F(s)$ is built. For every couple belonging to $F(s)$, the mean logarithmic contrast (22) associated to $F(s)$ is then:

$$
C(s)=\frac{1}{\# F(s)} \sum_{\left(x, x_{1}\right) \in F(s)} \min \left(\frac{|s-I(x)|}{\max (s, I(x))}, \frac{\left|s-I\left(x_{1}\right)\right|}{\max \left(s, I\left(x_{1}\right)\right)}\right)
$$

The best threshold $s_{0}$ verifies the following condition:

$$
s_{0}=\underset{s \in[0,255]}{\operatorname{argmax}} C(s)
$$

It is the threshold which has the best mean contrast along the associated border $F\left(s_{0}\right)$. Instead of using this method to binarize images, we use it to measure the contrast locally. The evaluated contrast equals $2 C\left(s_{0}\right)$ along the associated border $F\left(s_{0}\right)$.

\subsection{Experimental validation}

The methods have been tested using different video sequences as illustrated in Figs. 5, 6\&7 to present the three different applications. Samples of a more complicated sequence are shown in Fig. 10 where road scene enhancement is performed (see section 5.2). In this sequence, many interesting situations appear: curves, vegetation (trees, hedges), road signs, a cyclist, other cars. The proposed method copes quite well with these different situations. In particular, distant cars with low contrast are restored, especially in the first image.

\section{Conclusion}

In this paper, a framework for restoring the contrast of images grabbed from a moving vehicle has been presented. Contrary to usual methods where the scene structure is computed before restoring the contrast, we first compute the weather conditions and then we infer the scene structure which is refined during the restoration process. Due to our application context, the initial structure is based on a flat 


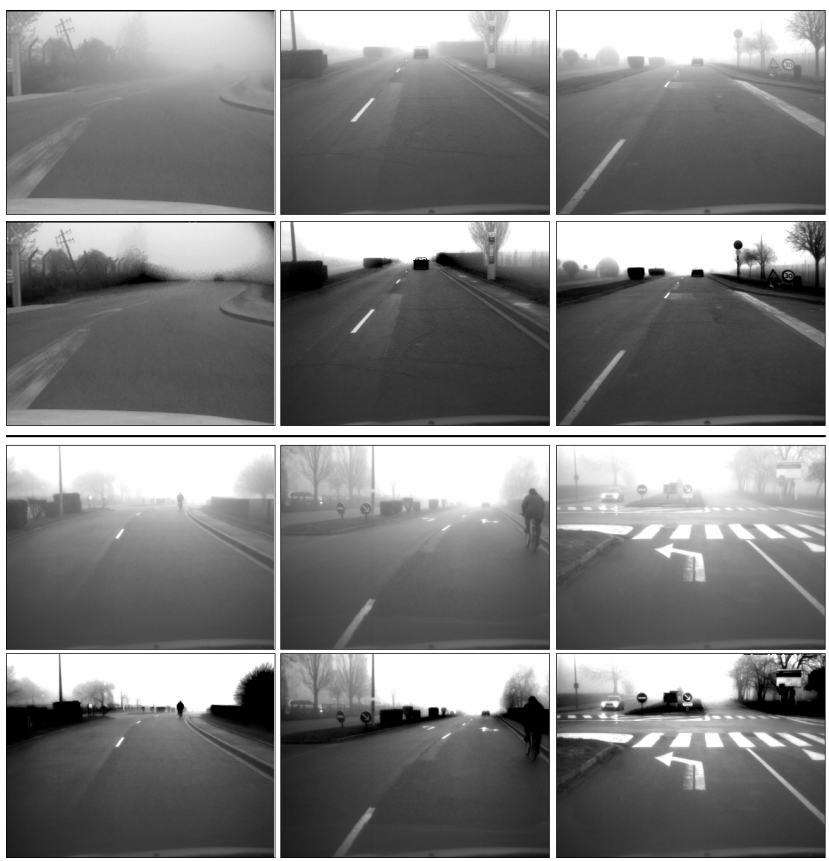

Figure 10. Example of contrast restoration on a video sequence implementing the road scene enhancement application. Row 1\&3: original images. Row 2\&4: corresponding restored images. Many situations appear: curves, vegetation, road signs, a cyclist, cars.

world assumption merged with some depth heuristics. To optimize the parameters of the scene, we proposed a metric of image quality based on a norm of local normalized correlation. We have applied our framework to three invehicle applications: road lane markings restoration, road scene enhancement and iterative road scene restoration, by fusing our restoration algorithm with a detection algorithm. Finally, results are assessed by computing the contrast of sample images before and after scene restoration.

\section{References}

[1] International lighting vocabulary. Number 17.4. Commission Internationale de l'Éclairage, 1987.

[2] E. Allard. Mémoire sur l'intensité et la portée des phares. 1876.

[3] P. Bhartia and I. Bahl. Millemeter Wave Engineering and Applications. John Wiley and Sons, 1984.

[4] P. Bouguer. Traité d'optique sur la gradation de la lumière. 1729.

[5] C. Bush and E. Debes. Wavelet transform for analyzing fog visibility. IEEE Int. Syst., 13(6):66-71, 1998.

[6] F. Cozman and E. Krotkov. Depth from scattering. In IEEE Conf. Computer Vision and Pattern Recognition, 1997.

[7] M. D. Grossberg and S. K. Nayar. Modeling the space of camera response functions. IEEE Trans. Pattern Analysis and Machine Intelligence, 26(10):1272-1282, 2004.

[8] N. Hautière, R. Labayrade, and D. Aubert. Real-Time Disparity Contrast Combination for Onboard Estimation of the
Visibility Distance. IEEE Trans. Intelligent Transportation Systems, 7(2):201-212, 2006.

[9] N. Hautière, J.-P. Tarel, J. Lavenant, and D. Aubert. Automatic Fog Detection and Estimation of Visibility Distance through use of an Onboard Camera. Machine Vision and Applications J., 17(1):8-20, 2006.

[10] T. M. Kwon. Atmospheric visibility measurements using video cameras: Relative visibility. Technical report, University of Minnesota Duluth, July 2004.

[11] R. Labayrade, D. Aubert, and J.-P. Tarel. Real time obstacle detection in stereovision on non flat road geometry through v-disparity representation. In IEEE Intell. Veh. Symp., 2002.

[12] J. Lavenant, J.-P. Tarel, and D. Aubert. Procédé de détermination de la distance de visibilité et procédé de détermination de la présence d'un brouillard. French patent 0201822 , LCPC / INRETS, 2002.

[13] J. McCall and M. Trivedi. Video-based lane estimation and tracking for driver assistance: survey, system, and evaluation. IEEE Trans. Intelligent Transportation Systems, 7(1):20-37, 2006.

[14] E. J. McCartney. Optics of the Atmosphere: Scattering by Molecules and Particles. 1975.

[15] W. Middelton. Vision through the atmosphere. University of Toronto Press, 1952.

[16] M. Minnaert. The Nature of Light and Color in the Open Air. 1954.

[17] S. G. Narashiman and S. K. Nayar. Interactive deweathering of an image using physical model. In IEEE Work. Color and Photometric Methods in Computer Vision, 2003.

[18] S. G. Narasimhan and S. K. Nayar. Vision and the atmosphere. Int. J. of Computer Vision, 48(3):233-254, 2002.

[19] S. G. Narasimhan and S. K. Nayar. Contrast restoration of weather degraded images. IEEE Trans. Patt. Anal. Machi. Intell., 25(6):713-724, June 2003.

[20] J. Oakley, B. Satherley, C. Harrison, and C. Xydeas. Enhancement of image sequences from a forward-looking airborne camera. In SPIE Image and Video Processing IV, volume 2666, pages 266-276, 1996.

[21] J. P. Oakley and B. L. Satherley. Improving image quality in poor visibility conditions using a physical model for contrast degradation. In IEEE Trans. Image Processing, number 7, pages 167-179, 1998.

[22] D. Pomerleau. Visibility estimation from a moving vehicle using the RALPH vision system. In IEEE Conf. Intelligent Transportation Systems, pages 906-911, 1997.

[23] Y. Schechner, S. Narasimhan, and S. Nayar. PolarizationBased Vision through Haze. Applied Optics, Special issue, 42(3):511-525, Jan 2003.

[24] Z. Wang, A. Bovik, and L. Lu. Why is image quality assessment so difficult? In IEEE Int. Conf. on Acoustics, Speech, and Signal Processing, volume 4, pages 3313-3316, 2002.

[25] Y. Yitzhaky, I. Dror, and N. Kopeika. Restoration of altmospherically blurred images according to weather-predicted atmospheric modulation transfer function. Optical Eng., 36, 1998. 